\title{
El uso de mapas conceptuales para la evaluación de los servicios de referencia virtual de las bibliotecas universitarias: una experiencia práctica
}

\author{
María Pinto * \\ Ramón A. Manso **
}

Artículo recibido:

18 de enero de 2012.

Artículo aceptado:

23 de jullio de 2012.

\section{RESUMEN}

La implantación de las tecnologías de la información proporciona a los servicios bibliotecarios cierto dinamismo en su concepción y desarrollo, como el caso del Servicio de Referencia Virtual (SRV), que además se debe evaluar con regularidad. El objetivo de este estudio es conocer la opinión de un grupo de profesionales, que trabajan en bibliotecas, participantes de clases sobre cómo ha de ser un SRV, qué elementos de calidad han de aportar y cuál ha de ser su proyección, desde un enfoque que contemple siempre al usuario como protagonista principal de dicho análisis. La metodología empleada es de corte cualitativo, basada en el uso del

* Universidad de Granada, España. mpinto@ugr.es

** Facultad de Ciencias de la Información y la Educación, UCLV, Cuba. manso@uclv. edu.cu

INVESTIGACIÓN BiBLIOTECOLÓGICA, Vol. 26, Núm. 57, mayo/agosto, 2012, México, ISSN: 0187-358X. pp. 249-269 
mapa conceptual como instrumento de diagnóstico y prospección a la vez que herramienta visual que permita observar y comparar gráficamente los criterios fundamentales aportados por los participantes en el análisis. Los resultados solicitan una mayor implicación del usuario, y la formación en habilidades informacionales desde el propio SRV.

Palabras Clave: Servicio de Referencia Virtual; evaluación; Mapas conceptuales; Servicio de Referencia Virtual; Usuarios; Evaluación de servicios.

\begin{abstract}
Concept maps as a way to evaluate virtual reference services in univerity libraries : a practical approach María Pinto and Ramón A. Manso

Implementing Information Technologies in library services requires some dynamic issues in its design and development, as the case of 'Virtual Reference Services' (VRS), which also must be evaluated on a regular basis. The aim of this study was to establish from the opinion of a group of librarians, participants in courses, about "how to build an VRS"; which elements are to provide quality and its projection, always from a perspective of users as the core of the analysis. The qualitative methodology is based on the use of 'concept maps' as a diagnosis and prospecting tools as well as a visual tool for observing and comparing graphically the basic criteria provided by the participants in the analysis. The results show that greater interaction with users is required as well as training in information skills from the VRS.
\end{abstract}

Keywords: Virtual Reference Service evaluation; Concept maps; Virtual Reference Service; Users; Library servicec assessment. 


\section{INTRODUCCIÓN}

T a idea de un servicio de referencia para ayudar a los usuarios desde un $ـ$ mostrador virtual, aprovechando las potencialidades ofrecidas por las nuevas tecnologías de la información y comunicación, surge en Estados Unidos durante la década de los 80 del siglo pasado, con la implementación de esta modalidad por la Biblioteca de Ciencias de la Salud de la Universidad de Maryland. En sus inicios la comunicación entre el servicio y los usuarios se realizaba mediante correo electrónico, facilitando así la reserva de documentos para su consulta, la solicitud de búsquedas automatizadas para la resolución de preguntas específicas y la fotocopia de artículos o libros (Weise, Borgendale, 1986). Los niveles de creatividad e innovación que las tecnologías de la información y comunicación han imprimido durante las últimas décadas al servicio de referencia tradicional permiten su evolución hacia las prestaciones más actuales. Hoy día el servicio de referencia virtual (SRV) se presta mediante una comunicación por ordenador entre el usuario y el proveedor del servicio. En este contexto la palabra referencia no tiene nada que ver con los conocidos trabajos de referencia, como son diccionarios y enciclopedias, sino más bien con el objetivo general de proporcionar "orientación" a los usuarios de las nuevas bibliotecas digitales. El concepto SRV incluye mecanismos de comunicación bidireccional entre el operador y el usuario, y la integración de productos y servicios para asegurar que la formación y la satisfacción de las necesidades informativas se satisfacen desde un solo interface de comunicación (Pinto, Manso, 2012).

Para que estos servicios de referencia virtual se conviertan en una herramienta eficaz es necesario conocerlos y evaluarlos de un modo continuo y permanente, pues tan solo así se puede mejorar la calidad de sus prestaciones.

\section{OBjetivos}

Muchas de las necesidades informativas básicas del estudiante que se mueve prioritariamente en los nuevos entornos digitales se suelen resolver satisfactoriamente mediante el uso de los SRV. Pero para que estos mantengan e incluso mejoren sus actuales prestaciones deben evolucionar de un modo permanente para adaptarse no sólo a las necesidades cambiantes de los usuarios, sino también a los continuos avances tecnológicos. Esto resulta trivial, pero por desgracia en las bibliotecas hay tendencia a diseñar, implementar y no rentabilizar el trabajo (Casey, Savastinuk, 2006). Por ello es necesario el desarrollo de mecanismos de evaluación de las prestaciones de los SRV, para tener 
un diagnóstico de la situación, poder valorar los aspectos positivos, detectar las principales deficiencias y tomar decisiones que conduzcan a la mejora. La evaluación continua de los servicios es considerada como una vía para la mejora de la calidad y la satisfacción del usuario (Rojas, 2003). El SRV, como toda oferta de información, no podría existir sin los usuarios, de modo que las percepciones que estos tengan sobre la prestación en concreto son fundamentales en cualquier evaluación que se pretenda realizar. El objetivo general de este estudio es conocer, desde la perspectiva de los profesionales de la información de los SRV, la valoración de diferentes aspectos relativos a la oferta de estos servicios virtuales, mediante el empleo de los mapas conceptuales como instrumento de diagnóstico. Esta herramienta estimula la reflexión y discusión sobre el objeto de estudio, permitiendo la organización del conocimiento y por consiguiente desvelar aspectos que sustentarán el rediseño de la oferta de información.

Entendemos que la aplicación de la técnica de los mapas conceptuales, como método para el análisis y recopilación de la información relacionada con el diseño y funcionamiento de los SRV, supone beneficios relacionados con el proceso de planificación, reflexión de sus prestaciones actuales y perspectivas de futuro. Esta técnica permite la asociación de conceptos que ayudan a develar a veces aspectos poco abordados, así como establecer relaciones entre dichos conceptos (Sherratt, Schlabach, 1990). El mapa conceptual ofrece la posibilidad de administrar el conocimiento a través de una sencilla pero estructurada red de conceptos, generando un producto gráfico del tipo representación de conocimiento. En realidad es un instrumento polivalente que permite la mejora de la eficiencia y eficacia del trabajo en equipo, la captación de conocimientos, la elaboración de entrevistas personales, la mejora de la productividad de las investigaciones e incluso la mejora de las relaciones entre colegas. Asimismo los mapas conceptuales constituyen una herramienta para la organización de la información personal, para su aplicación en la enseñanza y para el estudio de procesos (Novak, 1998). Este es el objetivo específico del presente estudio: desarrollar una mejor comprensión de los SRV y descubrir algunos aspectos que contribuyen a su mejora, tomando como instrumento de representación el mapa conceptual.

\section{Revisión DE LA LITERATURA}

Aunque el concepto de servicio de referencia virtual parece más bien difuso (Pace, 2003), una amplia definición los concibe como servicios de acceso a la información en los que el usuario hace preguntas empleando medios electrónicos 
(White, 2001). Pero las directrices y recomendaciones más o menos generalistas (IFLA, 2004) y/o específicas (RUSA, 2008) nos han permitido ampliar el conocimiento sobre estos servicios y descubrir algunos criterios para su evaluación así como los parámetros relacionados con las políticas del servicio y la interfaz de comunicación.

En todo caso debemos establecer una clara distinción entre las referencias sincrónicas (ocurren al mismo tiempo o velocidad) y asíncronas (no ocurren al mismo tiempo o velocidad). Por un lado, un servicio de referencia asíncrono permite a los bibliotecarios disponer del tiempo necesario para responder a las peticiones, lo que podría mejorar la precisión e integridad de las respuestas. Por otra parte, el propio canal asíncrono inhibe la aclaración de preguntas y carece de las señales no verbales y los comentarios sincrónicos que son cruciales en una entrevista de referencia eficaz, lo que puede aminorar la eficacia de la interacción usuario-bibliotecario (Shachaf, Horowitz, 2008).

Las evaluaciones de los SRV utilizan medidas similares a las empleadas en los servicios de referencia tradicionales: tipo de preguntas, precisión, exhaustividad, utilidad del servicio y satisfacción del usuario (Shachaf, Horowitz, 2008). La evaluación puede realizarse desde dos perspectivas: la del bibliotecario, y la del usuario que recibe la prestación (Pomerantz, 2008). Ambas perspectivas contemplan un conjunto de elementos que permiten identificar si dicha oferta satisface o no los requerimientos de información de la comunidad usuaria.

En esta investigación se ha optado por la perspectiva del usuario. En este sentido un estudio presentado por la Washington State Library (Hirko, 2004) ofrece una herramienta de evaluación práctica desde la perspectiva del usuario, basada en tecnología chat, con el fin de conocer cuáles son las mejores prácticas para el SRV. Otro trabajo interesante fue desarrollado en las universidades de Western Ontario y Toronto y se centra en las experiencias y percepciones de los usuarios en la consulta a los SRV. Los usuarios entrevistados realizaban primero un relato de su experiencia en el uso del servicio y después completaban una encuesta de satisfacción. Esta información era contrastada con las transacciones ocurridas durante la prestación del servicio, como fuente adicional de evidencia del proceso (Nilsen, 2005). De esta forma se detectaron ciertas deficiencias en el servicio y se propusieron soluciones de mejora.

Hay un tipo de servicio de referencia virtual, el colaborativo, que merece especial atención pues permite a los usuarios obtener ayuda para resolver sus necesidades de información a través de las bibliotecas que participan en el consorcio de referencia, aun cuando sus bibliotecas no estén disponibles 
(Kwon, 2005). Sin embargo, el traslado de un punto a otro en medio del proceso referencial puede frustrar a los usuarios con esta modalidad de servicio. De todos modos, los SRV del entorno universitario necesitan renovarse y evolucionar en consonancia con las nuevas tecnologías que permiten una retroalimentación más rica y una participación más activa de los usuarios. En esta línea de pensamiento el sistema debe incorporar las nuevas tendencias en tecnologías de la información derivadas de la Web 2.0, que permiten a los usuarios participar y compartir a un nivel sin precedentes.

Como procedimiento innovador para recoger la voz del usuario hemos empleado las potencialidades de los mapas conceptuales, utilizados preferentemente como una herramienta de aprendizaje y de evaluación para cualquier modelo de conocimiento, ya sea declarativo o procedimental. Se trata de un valioso instrumento que posibilita un tipo de evaluación estructural centrado en el aspecto del mapa y de sus enlaces (Pottier et al., 2010).

Los mapas conceptuales han sido utilizados como instrumento de apoyo para el aprendizaje en muchas disciplinas académicas: en ciencias de la vida destacan las investigaciones de Briscoe y LaMaster (1991) y Kinchin (2001) sobre el impacto de los mapas conceptuales en la enseñanza de la biología; los trabajos de Novak (1990, 1998); Hilbert y Renkl (2008), entre otros, aplicados al área de educación; Hsu y Hsieh (2005) estudiaron su repercusión entre los estudiantes de enfermería; Brandt et al. (2001) analizaron sus efectos en los estudios de química; Tümen y Taspmar (2007) los emplearon en la enseñanza de lengua, mientras que Markha, Mintzes y Jones (1994) lo usaron como una herramienta de investigación y evaluación. Todos los autores llegaron a la conclusión de que los mapas conceptuales son un método de representación gráfica de la información, que afecta positivamente a la tasa de éxito en los resultados académicos del estudiante, pues reducen los problemas de sobrecarga de información y mejoran la desorientación conceptual en el aprendizaje (Chen, Kinshuk y Chen, 2008). Por tanto, los mapas conceptuales pueden emplearse como una herramienta eficaz de enseñanza, para organizar los contenidos de un curso, preparar las clases o presentar el material a los estudiantes (Hilbert y Renk1 2008); como un instrumento de aprendizaje para los estudiantes, que les ayude a tomar, organizar y sistematizar las notas de clase, mejorando la retención de las ideas fundamentales (O'Donnell, Dansereau y Hall, 2002), y la resolución de problemas en clase (Pottier, et al., 2010, Barbosa, Ramos, 2006); y como una herramienta de evaluación, enfocada a evaluar el aprendizaje del estudiante y el proceso docente, ofreciendo retroalimentación tanto al profesor como al estudiante, para valorar aspectos relacionados con la comprensión de los conceptos, determinando aquellos conceptos erróneos que necesitan una mayor clarificación. En este sentido, 
afirma Kaya (2008) que son múltiples los beneficios del uso de los mapas conceptuales, pues favorecen los procesos de docencia-aprendizaje, mejoran las habilidades de selección y comprensión conceptual y ayudan a la toma de decisiones.

Pinto, Doucet y Fernández (2010) proponen una metodología para analizar y medir las habilidades y destrezas de los estudiantes universitarios en la creación de mapas conceptuales, como herramienta para diagnosticar y mejorar la formación de las competencias de análisis, síntesis, organización y representación de la información. Los estudiantes ponen de manifiesto las escasas competencias que tienen sobre este tema, debido a que no hay oferta formativa en el currículo sobre técnicas de representación gráfico-conceptual de la información.

En el ámbito de las bibliotecas escasean los estudios sobre el uso de los mapas conceptuales en los procesos y servicios de referencia. Pero hay algunos referentes representativos, como Sherratt y Schlabach (1990), que abordan el uso de los mapas conceptuales elaborados por estudiantes de posgrado en un curso de referencia y servicios de información. De igual forma, resaltan la importancia que tiene para los bibliotecarios la aplicación de esta herramienta en el servicio de referencia, la enseñanza y la investigación. De interés es la experiencia desarrollada por la Biblioteca Juan Roa Vásquez de la Universidad El Bosque, que emplea el uso de los mapas conceptuales para la gestión del servicio de referencia, como forma de agregar valor al servicio y como técnica para documentar todo el proceso que el bibliotecario sigue en la resolución de una demanda de información (Vargas, 2010).

En cualquier caso, el uso del mapa conceptual como vía para evaluar el SRV desde la perspectiva del usuario, y de esta forma poder recopilar información y sugerencias para el diseño y desarrollo de estos servicios, parece un asunto novedoso e interesante.

\section{Metodología}

La metodología empleada es fundamentalmente cualitativa, basada en la aplicación de las técnicas de brainstorming, focus group y la expresión del pensamiento en voz alta, con la finalidad de recabar información sobre el SRV para posteriormente utilizar el mapa conceptual como instrumento de representación documental y visualización gráfica de la información. La técnica de focus groups se empleó para conocer la opinión de los usuarios sobre la evaluación de los SRV, contribuyendo a la participación y el intercambio de experiencias en el grupo (Whitlatch, 2001). Constituye una forma eficiente 
de obtener información sobre experiencias, pensamientos, preocupaciones y valores de los usuarios (Kuruppu, 2007).

Para este estudio se utilizó una muestra por conveniencia, dirigida y no probabilística (Hernández Sampieri, Fernández, Baptista, 2010), conformada por siete estudiantes matriculados en el curso "Evaluación y Calidad en Bibliotecas Universitarias" que forma parte del Master en Información Científica: Tratamiento, Acceso y Evaluación, de la Universidad de Granada. La mayoría de los participantes son titulados en biblioteconomía y otros son profesionales que trabajan en bibliotecas y servicios de información especializados.

Después de varias sesiones teórico-prácticas sobre temas emergentes de evaluación y calidad en los servicios de biblioteca, se abordó la calidad del servicio de referencia virtual, incidiendo en la gestión del cambio ante los avances tecnológicos y las necesidades y expectativas de las nuevas generaciones de usuarios. La sesión formativa (de dos horas de duración) se realizó en junio de 2010 y se articuló en tres fases.

- La sesión presencial, en la que un experto en la materia ofreció una visión panorámica de los SRV. Se invitó a los asistentes a que participaran en una tormenta de ideas sobre aspectos relacionados con la organización del servicio, formas de comunicación, profesionales que lo suministran, etcétera.

- El focus group, que empleó este protocolo de preguntas semiestructuradas:

- ¿Qué retos significativos crees que debe afrontar el profesional de la información en los SRV?

- ¿Consideras necesario integrar los servicios de referencia en un solo punto de acceso y en las redes sociales?

- ¿El usuario debe formar parte en el proceso de diseño y desarrollo del servicio de referencia virtual?

- ¿Crees que el SRV actual tiene dificultades de accesibilidad y visibilidad para el usuario que pueden dificultar su uso?

A fin de validar la idoneidad y la consistencia de las preguntas del focus group se realizó un pre-test con un grupo de expertos académicos y bibliotecarios de las Universidades de Granada y de Central de Las Villas, incorporándose alguna mejora en la formulación del último ítem. El contenido de cada una de estas preguntas fue expresado por los participantes en la sesión de trabajo en forma de idea que reflejara y sintetizara sus puntos de vista con relación a la forma de presentar la oferta en la actualidad y su percepción sobre el futuro de los SRV, para sobrevivir y seguir respondiendo a las necesidades e intereses de la comunidad usuaria. 
Se tomó nota de todos los comentarios, se grabó la sesión de trabajo, se procedió al análisis de contenido, identificando y agrupando los conceptos de forma que se pudieran categorizar.

- La representación conceptual de las principales ideas sobre cómo debía ser un SRV, por medio de mapas conceptuales. Una vez identificados los conceptos y agrupados en categorías y su redacción en forma de idea, cada participante en la sesión de trabajo procedió a elaborar un mapa conceptual desde su propia perspectiva, tanto desde el punto de vista de lo que como profesional podía añadirle al servicio, como de lo que como usuario desearía recibir. Los participantes hicieron los mapas manualmente y después se pasaron a soporte digital usando el programa Inspiration 7.6.

\section{Resultados}

A partir de la tormenta de ideas (brainstorming) se listaron y agruparon una serie de conceptos que son clave en el entorno de los SRV: accesibilidad, usuarios, promoción, cooperación, diseño, comunicación y formación (Tabla 1). Cada una de estas categorías da respuesta a las diferentes interrogantes que se plantearon para suscitar el debate entre los participantes.

Tabla 1. Opiniones, categorías y propuestas plasmadas durante la sesión de brainstorming

\begin{tabular}{|c|c|c|}
\hline Opiniones & Categorías & Propuestas \\
\hline $\begin{array}{l}\text { - Deficiencia en la accesibilidad. } \\
\text { - Unificar puntos de acceso al ser- } \\
\text { vicio. } \\
\text { - Acceso total. } \\
\text { - Accesos integrados. }\end{array}$ & Accesibilidad & $\begin{array}{l}\text { Creación de una página web visual- } \\
\text { mente atractiva para los usuarios que } \\
\text { integre otros productos y servicios. }\end{array}$ \\
\hline $\begin{array}{l}\text { - Implicación del usuario. } \\
\text { - Satisfacción del usuario. } \\
\text { - Necesidades de los usuarios } \\
\text { - Adecuarse al segmento de usua- } \\
\text { rios que se dirige. }\end{array}$ & Usuarios & $\begin{array}{l}\text { Adecuar el servicio a las necesida- } \\
\text { des del usuario, implicándolo en su } \\
\text { diseño y desarrollo. }\end{array}$ \\
\hline $\begin{array}{l}\text { - Se debe promocionar lo que se } \\
\text { ofrece. } \\
\text { - Marketing de la información. } \\
\text { - Introducir la biblioteca en las redes } \\
\text { sociales. }\end{array}$ & Promoción & $\begin{array}{l}\text { Promoción del servicio de referencia } \\
\text { virtual a través de diferentes medios } \\
\text { y a todos los niveles. }\end{array}$ \\
\hline $\begin{array}{l}\text { - Usuario como cooperador en el } \\
\text { servicio y no como simple usuario. } \\
\text { - Participación. } \\
\text { - Inteligencia colectiva. }\end{array}$ & Cooperación & $\begin{array}{l}\text { Participación activa del usuario en } \\
\text { el diseño y desarrollo del servicio de } \\
\text { referencia virtual. }\end{array}$ \\
\hline
\end{tabular}




\begin{tabular}{|c|c|c|c|}
\hline$>$ & $\begin{array}{l}\text { - Identificación de debilidades. } \\
\text { - Identificar prioridades. } \\
\text { - Organización conjunta del servicio } \\
\text { entre bibliotecarios y usuarios. } \\
\text { - Introducción de mejoras a partir } \\
\text { del estudio de la satisfacción del } \\
\text { usuario. } \\
\text { - Políticas de evaluación - calidad } \\
\text { enfocadas al usuario. }\end{array}$ & Diseño & $\begin{array}{l}\text { Identificación de debilidades y prio- } \\
\text { ridades que tengan en cuenta la } \\
\text { satisfacción del usuario, así como la } \\
\text { organización del servicio y estable- } \\
\text { cimiento de políticas de evaluación } \\
\text { que hayan sido elaboradas previa co- } \\
\text { laboración de la comunidad usuaria. }\end{array}$ \\
\hline & $\begin{array}{l}\text { - Comunicación bidireccional. } \\
\text { - Foros de usuarios del servicio. } \\
\text { - Charlas online para evacuar dudas } \\
\text { concretas. }\end{array}$ & Comunicación & $\begin{array}{l}\text { Implementación de canales de co- } \\
\text { municación que faciliten la interac- } \\
\text { ción entre usuarios y bibliotecarios } \\
\text { de manera bidireccional. }\end{array}$ \\
\hline & $\begin{array}{l}\text { - Diálogo online entre bibliotecario } \\
\text { y usuario. } \\
\text { - Alfabetización informacional. } \\
\text { - Trabajo conjunto online. } \\
\text { - Tutoriales interactivos. } \\
\text { - Guías online. }\end{array}$ & Formación & $\begin{array}{l}\text { Potenciar desde el servicio de refe- } \\
\text { rencia virtual la formación en habili- } \\
\text { dades de información. }\end{array}$ \\
\hline
\end{tabular}

Entre los aspectos más significativos que se pueden señalar de esta parte de la sesión de trabajo y que se corroboran posteriormente al visualizar gráficamente los mapas conceptuales, sobresalen los siguientes: la necesidad de incorporar al SRV los nuevos avances tecnológicos que posibiliten tanto una mayor accesibilidad como su integración y promoción; la necesidad de una mayor retroalimentación con el usuario, potenciando su participación en el diseño de la oferta (Casey, Savastinuk, 2006), (Maness, 2006), (O'Reilly, 2005), (OCLC, 2004); finalmente, la importancia de la formación en alfabetización informacional en el desarrollo de los SRV, enfocada a profesionales y usuarios (Beck, Turner, 2001), (Ellis, 2004), (Hull, 2004), (Johnston, 2003).

La representación gráfica mediante el empleo de los mapas conceptuales para el análisis del SRV ha permitido visualizar las redes conceptuales de los participantes, mostrando las diferentes formas de ver un mismo problema, según la perspectiva de cada usuario, pero asimismo destacando los puntos coincidentes. La presentación de los mapas responde al formato conocido como "araña", aunque con distintos grados de complejidad según el autor. De este modo cualquier comparación entre ellos parece más fácil de abordar.

El Mapa conceptual 1 plantea el paralelismo entre el SRV y la interfaz integrada que permite su desarrollo y evolución. Desde la planificación estratégica, el servicio se basa en cuatro pilares: la alfabetización informacional de personal y usuarios, las redes de información, el apoyo de servicio a los profesores y la difusión. El concepto de interfaz integrada incluye aspectos relacionados con horas de apertura, integración de los recursos, canales de comunicación y facilidad de acceso (Fig. 1). Se visiona el servicio desde un enfoque estratégico, 
funcional y acorde con las tecnologías emergentes y el nuevo paradigma de la alfabetización informacional, reafirmado la tesis de Wong (2010) al plantear que la formación es un elemento crucial para la efectividad del servicio y su calidad.

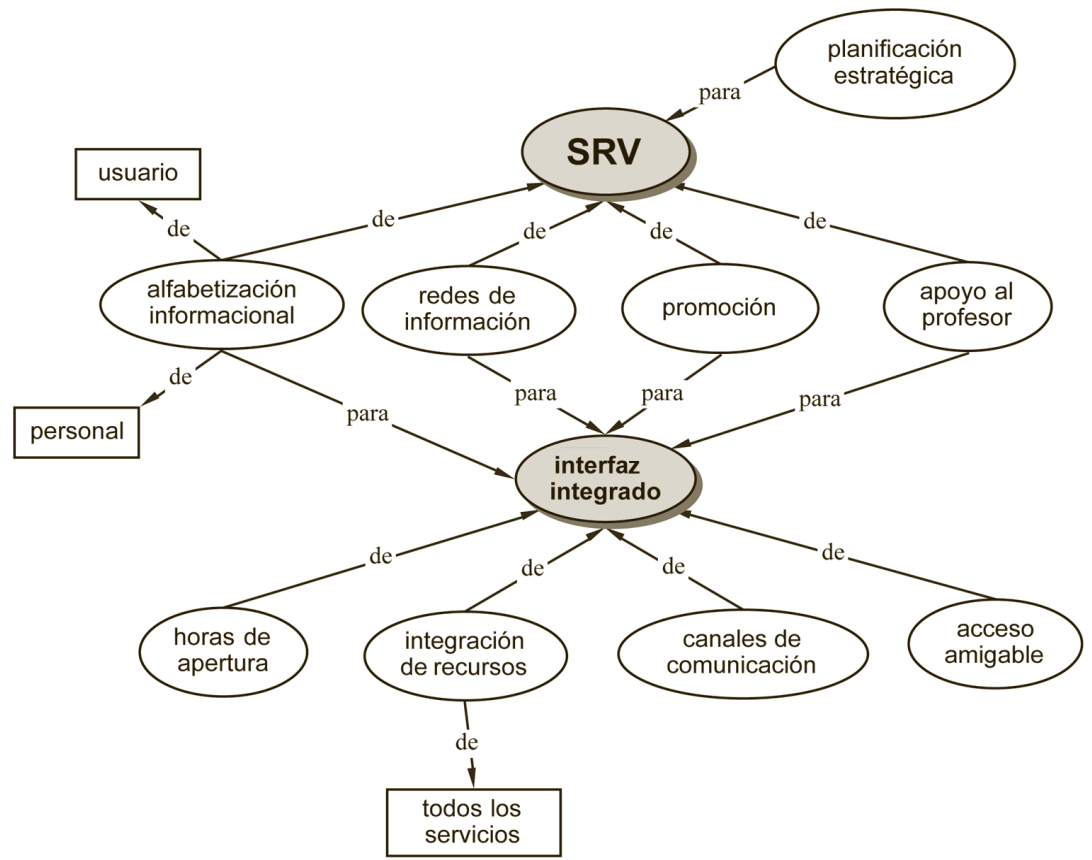

Fig. 1. Mapa conceptual 1

El concepto de acceso a la información, planteado desde el doble aspecto del usuario del servicio y del bibliotecario, fundamenta la concepción del Mapa conceptual 2. Entre estos dos polos se establece una comunicación y colaboración bidireccional. Desde la perspectiva del usuario, el acceso a la información debe ser satisfactorio, atractivo, integrado y accesible. El bibliotecario debe promover, diseñar, evaluar y planificar tal acceso a la información. El establecimiento de una retroalimentación permanente entre estos dos componentes del SRV contribuirá en gran medida a elevar la calidad de la oferta, teniendo siempre presente la formación continua de usuarios y bibliotecarios (Fig. 2). Este enfoque es más bien social, encaminando todas sus actuaciones hacia el acceso a la información y la satisfacción del usuario. 


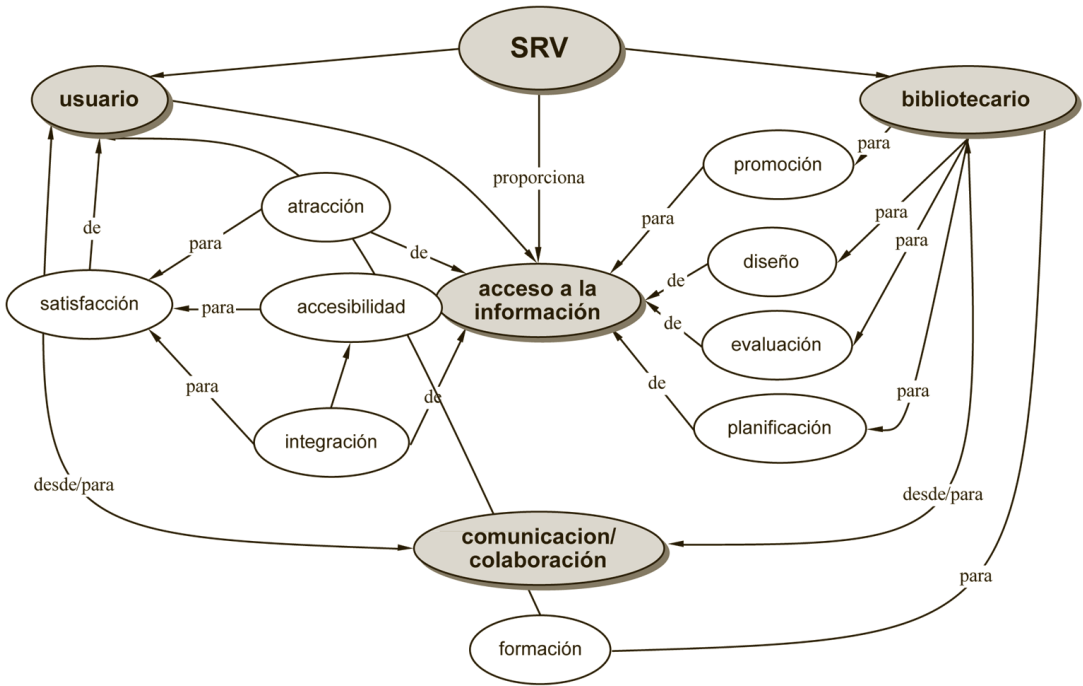

Fig. 2. Mapa conceptual 2

El Mapa conceptual 3 representa como conceptos nucleares del SRV la trilogía usuario-bibliotecario-recursos. El usuario debe ser un agente activo en relación con el servicio, participativo, implicado, y con la capacitación adecuada. El bibliotecario debe poner en juego todas sus estrategias pedagógicas. El servicio centra sus prioridades en las horas de apertura, la mejora continua, la campaña de promoción y la interfaz, para que éste sea lo más atractivo posible (Fig. 3). Por tanto, la representación conceptual de este mapa se encuadra en el enfoque estratégico ya mencionado.

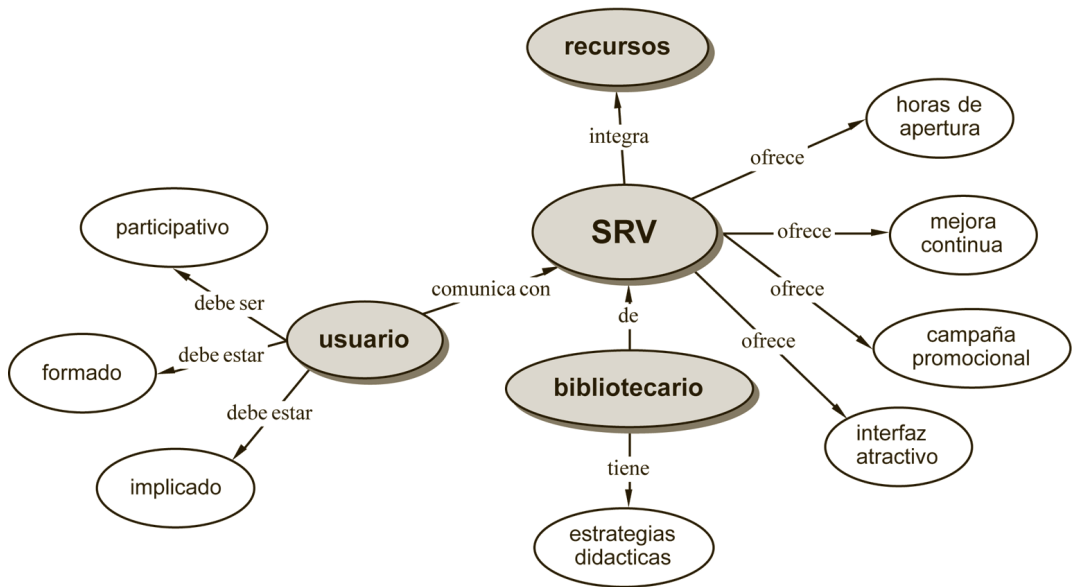

Fig. 3. Mapa conceptual 3 
También el Mapa conceptual 4 registra en primer término la interacción bidireccional del bibliotecario con el usuario, siendo éste un elemento clave a tener presente en el diseño atractivo del servicio. Destaca el concepto de política bibliotecaria para el diseño, evaluación y mejora continua del SRV, orientado a facilitar la recuperación y acceso de la información. Otro componente clave es la formación en competencias informacionales de los usuarios, planificada y asistida por el bibliotecario (Fig. 4). En síntesis, se plantea una visión social del SRV, destacando aspectos claves relacionados con la formación en alfabetización informacional y el uso de estrategias didácticas.

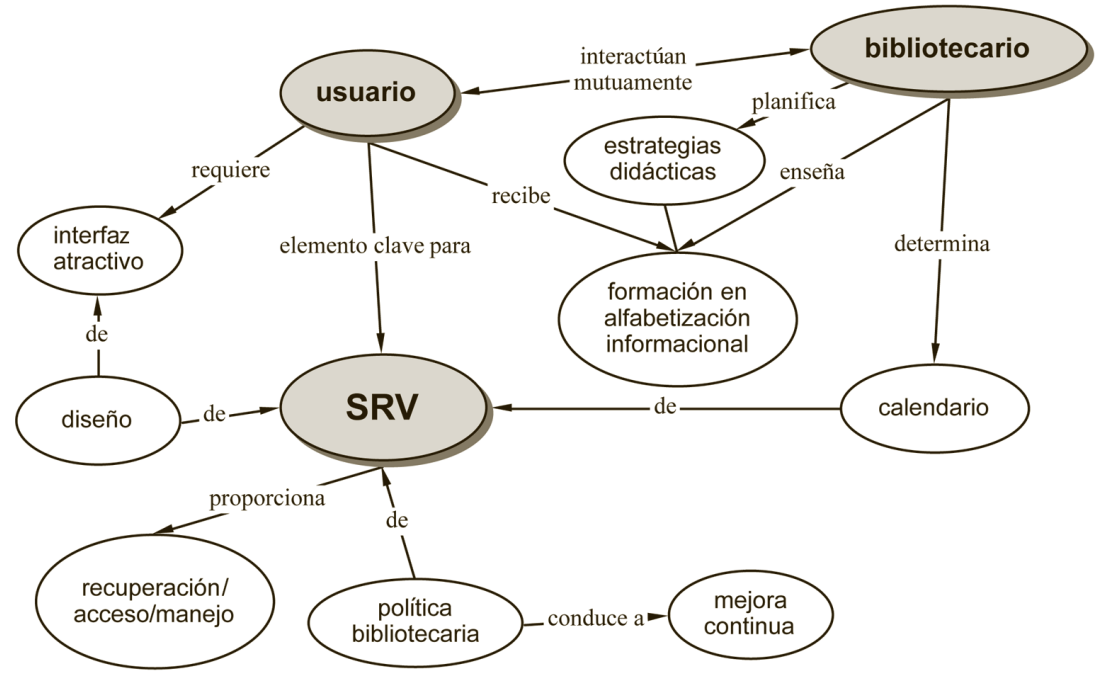

Fig. 4. Mapa conceptual 4

En el Mapa conceptual 5 se visualiza el conjunto de componentes clave que articulan el funcionamiento de un SRV: diseño, política bibliotecaria, promoción, canales de comunicación, formación, usuario y recursos. Del análisis del mapa se infiere que todo SRV ha de pasar por un proceso de planificación acorde con las políticas de calidad de la institución, incorporando la formación en competencias informacionales de usuario y bibliotecario. Otros conceptos planteados son: el uso de los canales de comunicación, destacando el chat, la videoconferencia, las redes sociales y el teléfono; los recursos y sus facilidades de acceso; la recuperación y gestión; y todo lo concerniente al diseño del servicio, que debe ser visualmente atractivo, amigable y fácil de usar (Fig. 5). Por consiguiente, estamos ante un enfoque estratégico del SRV, marcado especialmente por la promoción y el uso de canales innovadores de comunicación como las redes sociales. 


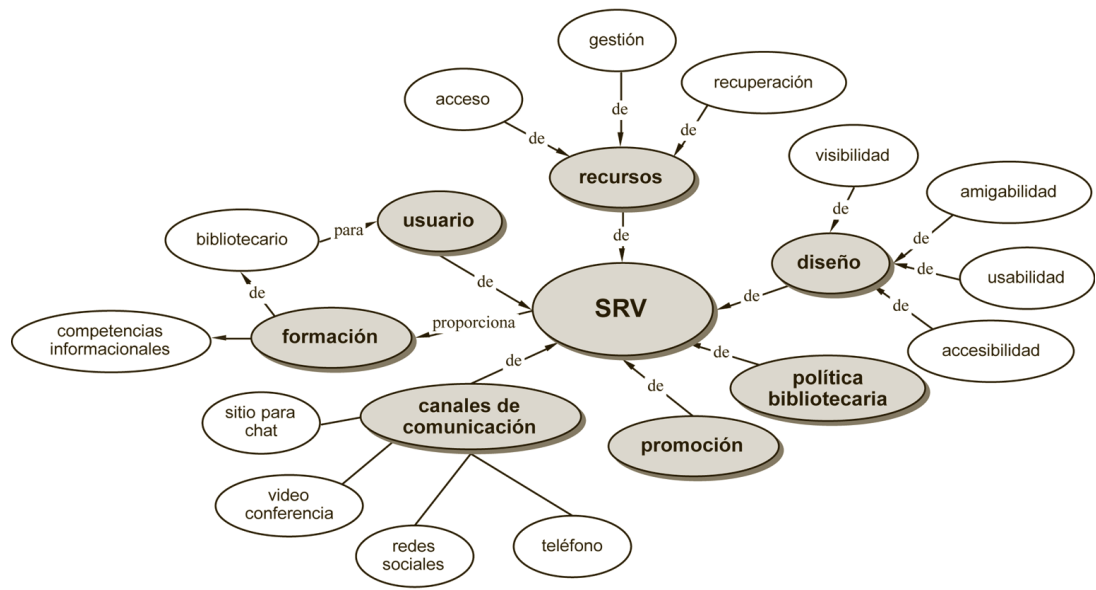

Fig. 5. Mapa conceptual 5

El Mapa conceptual 6 destaca la información, el usuario y su comunicación con el bibliotecario como elementos fundamentales en el desarrollo de los SRV. Se aportan conceptos relacionados con el acceso, manejo y recuperación de información. También forman parte del servicio la alfabetización informacional y la incorporación de los elementos formativos mediante tutoriales (Fig. 6). Esta visión encaja en el enfoque social del SRV, marcado por el protagonismo del usuario, la comunicación bidireccional con el bibliotecario y el uso de estrategias y herramientas para el entrenamiento en competencias informacionales.

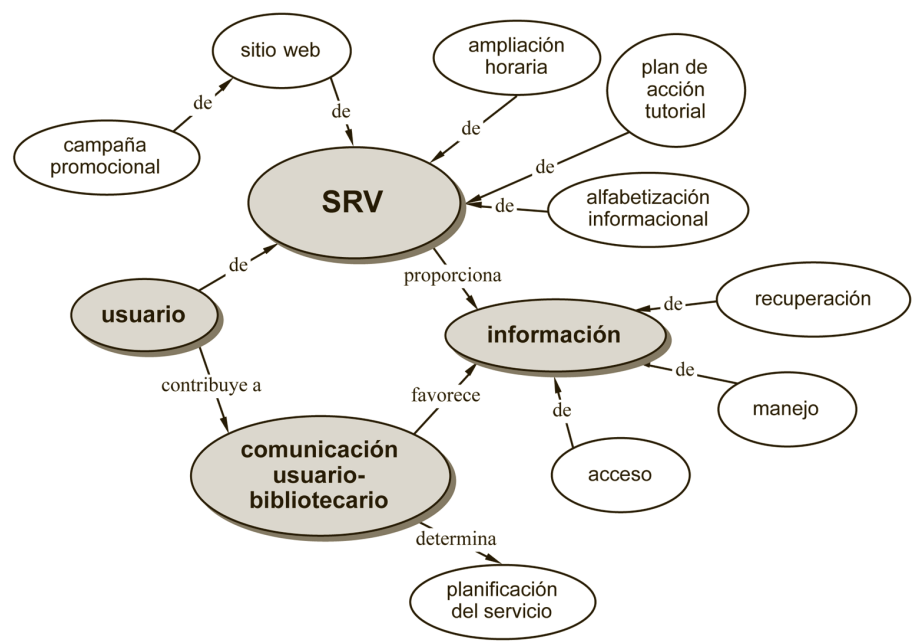

Fig. 6. Mapa conceptual 6 
Por último, en el Mapa conceptual 7 el SRV se despliega en ocho conceptos fundamentales: diseño, recursos, accesibilidad, usuario, servicio, comunicación, promoción y colaboración. El rol del usuario como agente en el proceso de referencia, su participación y cooperación en el desarrollo del mismo, la evaluación del servicio conectada a la satisfacción del usuario, la implementación de nuevos canales de comunicación, la presencia de la oferta del servicio en las redes sociales como vía de promoción y la accesibilidad son algunas de las cuestiones planteadas (Fig.7). De nuevo, esta red de conceptos representados en el mapa conceptual nos acerca a un enfoque estratégico del SRV, que prioriza el diseño, los recursos, la colaboración, la comunicación y al usuario.

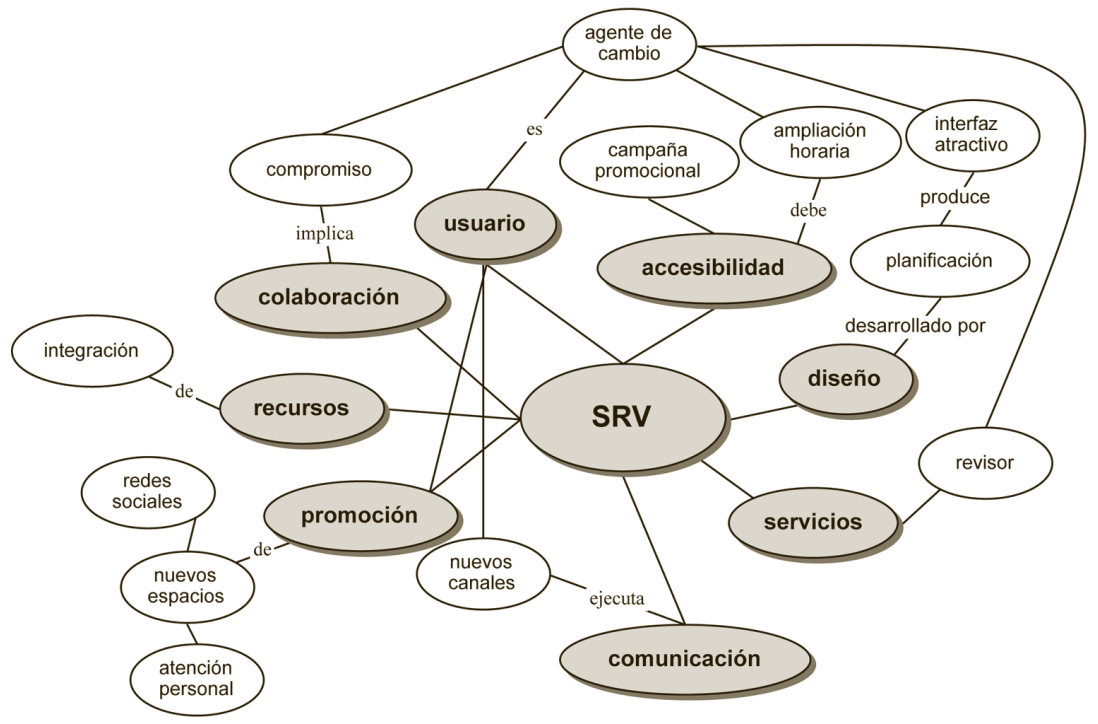

Fig.7. Mapa conceptual 7

Del análisis realizado se desprende que el SRV, como punto de convergencia entre usuarios y bibliotecarios, ha de ir evolucionando hacia nuevos modelos que privilegien la participación de la comunidad usuaria en su diseño y desarrollo, convirtiéndose en un espacio integrado que combine diferentes ofertas y propicie la comunicación entre todos los actores. Los conceptos más representados por los participantes aparecen estratificados en orden de importancia (Fig. 8). El concepto principal, en torno al cual giran los SRV, es el de usuario, razón de ser, origen y destino de estos singulares servicios de información. En realidad la satisfacción del usuario es el objetivo primordial de todo servicio, una idea profundamente enraizada en el pujante paradigma de la calidad. En el 
segundo estrato conceptual se encuentran las nociones de alfabetización informacional, accesibilidad y promoción. Ello viene a confirmar la estrecha relación entre los SRV y la alfabetización informacional de proveedores (bibliotecarios) y usuarios. La mayor cualidad exigible a un SRV es su fácil acceso. También ratifica que estos servicios se fundamentan en el interés por su promoción, tanto de las instituciones implicadas como de los propios usuarios, reflejando la conciencia que existe de la necesidad de promocionar estos SRV entre la población universitaria. En una tercera cota aparece el concepto de biblioteca, que es la parte esencial del servicio. En el cuarto nivel se sitúa el concepto comunicación, referido a la necesidad de un diseño permeable tanto para los emisores como para los receptores de la información. Un quinto grupo conceptual ofrece una diversidad de conceptos (recursos, horario, planificación, interfaz, diseño, canales, atractivo) que reflejan algunas de las cualidades necesarias para la planificación de un SRV de calidad, según los participantes. También los estratos seis, siete y ocho sugieren indicadores interesantes a tener en cuenta en el diseño e implementación de los SRV: estrategias didácticas, recuperación, redes sociales, amigabilidad, colaboración, información, manejo, política, gestión, tutoriales, usabilidad, visibilidad, web.

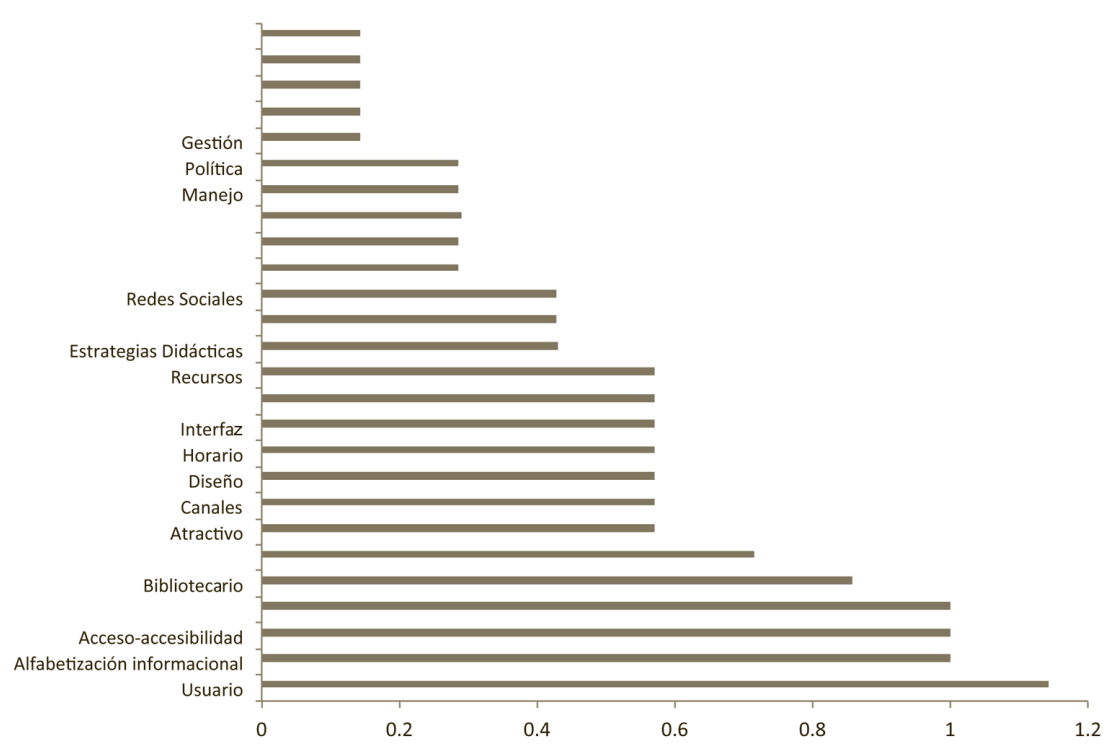

Fig. 8. Conceptos SRV estratificados por su importancia 
En resumen, del análisis de contenido de estos mapas conceptuales se desprende que la visión que aportan los participantes está en línea con las contribuciones de Agosto, et al (2011), quienes plantean la necesidad de que el servicio de referencia virtual, con una creciente implicación del usuario, camine hacia un modelo basado en la filosofía 2.0, fomentando así la retroalimentación y la participación. De igual forma, destaca la escasa promoción de estos servicios, siendo uno de los puntos débiles, unido a los problemas tecnológicos y a la falta de indicaciones de uso y sistemas de ayuda, por lo que resulta importante que los SRV aprendan a hacer un uso eficaz de los mecanismos de comunicación, a fin de contrarrestar estos efectos negativos (Nilsen, Ross, 2006).

Por otro lado, como afirma Mu et al. (2011), la escasa utilización de los SRV se ha convertido en un desafío para las bibliotecas, que deben buscar todas las vías posibles para aminorar la brecha existente entre los usuarios y el servicio, apostando por un diseño amigable, sencillo e intuitivo, que esté dotado de mecanismos de accesibilidad efectivos, como la inclusión de textos y gráficos llamativos en los sitios web de la biblioteca, la reubicación del enlace al servicio en un lugar visible, o el empleo de etiquetas de textos más fáciles de usar.

\section{CONCLUSIONES Y RECOMENDACIONES}

A modo de síntesis se infiere que, según los participantes, los indicadores fundamentales para el diseño y desarrollo de un servicio de resumen son los siguientes: el protagonismo del usuario, en todas sus facetas (participación, colaboración, formación, ...); la importancia de la alfabetización informacional como elemento innovador del servicio que mejore las competencias informacionales de bibliotecarios y usuarios; la promoción y accesibilidad del servicio; la biblioteca y el bibliotecario como agentes y vehículos del servicio, y la comunicación entre todos los agentes y canales implicados, incluidas las redes sociales.

Los resultados obtenidos contribuyen a enriquecer la literatura sobre el tema de los SRV y sus modos de evaluación centrados en el usuario. Pero sobre todo nos permiten derivar algunas recomendaciones que puedan ser tenidas en cuenta en la planificación de esta modalidad de servicio bibliotecario:

- Acceso integrado a todos los productos y servicios.

- Implicación y participación del usuario en el proceso de creación de 
productos y servicios de información como estrategia de mercado (Pinto, Manso, 2012).

- Promoción del SRV a través de diferentes canales de comunicación.

- Cooperación entre diferentes instituciones y del propio usuario.

- Mejora de los canales de comunicación bidireccional entre bibliotecarios y usuarios.

- Diseño coherente, atractivo, sencillo de la interfaz del servicio, que potencie la accesibilidad de cualquier persona.

- Formación en alfabetización informacional, con especial énfasis en el entrenamiento de las competencias informacionales, no solo del usuario sino también del propio bibliotecario.

En definitiva, resulta evidente que la evaluación de los servicios de información, y en este caso particular del SRV a partir del enfoque centrado en el usuario y mediante el empleo de la técnica de los mapas conceptuales, nos ha permitido detectar áreas de interés y predecir los necesarios cambios y adecuaciones del servicio de referencia virtual, con el propósito de satisfacer las demandas y las expectativas de los usuarios.

\section{Bibliografía}

Agosto, D. E. et al. (2011), "A Model of the Reference and Information Service Process: An Educators' Perspective”, Reference \& User Services Quarterly, Vol. 50, No. 3, pp. 235-44.

Barbosa, J., Ramos, F. (2006), "Mapas conceptuales como medio para la recuperación, gestión e intercambio de fuentes de información de apoyo al aprendizaje en plataformas de e-learning", in A. J.; Novak Cañas, J. D. (Ed.), Concept Maps: Theory, Methodology, Technology, San José, Costa Rica.

Beck, S. E., Turner, N. B. (2001), "On the Fly BI: Reaching and Teaching from the Reference Desk", The Reference Librarian, Vol. 34 No. 72 , pp. 83-96.

Brandt, L. et al. (2001), The impact of concept mapping and visualization on the learning of secondary school chemistry students, International Journal of Science Education, Vol. 23, No. 12, pp. 13031313.

Briscoe, C.; LaMaster, S. (1991), Meaningful learning in college biology through concept mapping, The American Biology Teacher, Vol. 53, No. 4, pp. 214-229.

Casey, M. E., and Savastinuk, L. C. (2006), "Library 2.0: Service for the next-generation library", Library Journal, no. 14, available at: <http:// www.libraryjournal.com/article/CA6365200.html> (21-9-2010). 
Chen, N.; Kinshuk, W.C.; Chen, H. (2008), Mining e-Learning domain concept map from academic articles, Computers E Education, Vol. 50, No. 3, pp. 1009-1021.

Ellis, L. A. (2004), "Approaches to Teaching through Digital Reference", Reference Services Review, Vol. 32 No. 2, pp. 103-19

Hernández, Sampieri, R.; Fernández Collado, C.; Baptista Lucio, P. (2010). Metodología de la Investigación, 4ta ed. México: McGraw Hill

Hilbert, T.S.; Renkl, A. (2008), Concept mapping as a follow-up strategy to learning from texts: what characterizes good and poor mappers? Instructional Science, Vol. 36, No. 1, pp. 53-73.

Hirko, B. (2004), veT: The Virtual Evaluation Toolkit, Washington State Library Olympia, WA.

Hull, C. M. "An Analysis of Information Literacy Instruction in the Virtual Reference Service of a Public Library System." University of North Carolina, 2004.

Hsu, L.; Hsieh, S. (2005), Concept maps as an assessment tool in a nursing course, Journal of Professional Nursing, Vol. 21, No. 3, pp. 141-149.

IFLA (2004), "Recomendaciones para el Servicio de Referencia Digital", available at: http://www.ifla.org/VII/s36/pubs/drg03-s.htm (30-1-2011).

Johnston, P. E. (2003), "Digital reference as an instructional tool: Just in time and just enough", Searcher, Vol. 11 No. 3, pp. 31-33.

Kaya, O.N. (2008), "A Student-centred Approach: Assessing the Changes in Prospective Science Teachers' Conceptual Understanding by Concept Mapping in a General Chemistry Laboratory", Research in Science Education, Vol. 38, pp. 91-110.

Kinchin, I.M. (2001), If concept mapping is so helpful to learn biology Why aren't we all doing it? International Journal of Science Education, Vol. 23, No. 12, pp. 1257-1269.

Kuruppu, P. U. (2007), "Evaluation of Reference Services-A Review", The Journal of Academic Librarianship, Vol. 33 No. 3, pp. 368-81.

Kwon, N. (2005), "User satisfaction with referrals at a collaborative virtual reference service", Information Research, Vol. 11, No. 2.

Maness, J. M. (2006), "Library 2.0 theory: web 2.0 and its implication for libraries", Webology, no. 2, available at: <http://www.webology.ir/2006/v3n2/a25.html> (25-6-2010).

Markha, K.M.; Mintzes, J.; Jones, G.M. (1994), The concept map as a research and evaluation tool: further evidence of validity, Journal of Research in Science Teaching, No. 31, pp. 91-101.

$\mathrm{Mu}, \mathrm{X}$ et al. (2011), "A Survey and Empirical Study of Virtual Reference Service in Academic Libraries", The Journal of Academic Librarianship, Vol. 37, No. 2, pp. 120-129 
Nilsen, K. "Virtual versus Face-to-Face Reference: Comparing Users' Perspectives on Visits to Physical and Virtual Reference Desks in Public and Academic Libraries." Paper presented at the World Library and Information Congress: 71th IFLA General Conference and Council, "Libraries - A voyage of discovery", Oslo, Norway, August, 14-18 2005.

Nilsen, K.; Ross, C.S. (2006), "Evaluating Virtual Reference from The Users' Perspective,” The Reference Librarian, No. 95/ 96, pp. 53-79.

Novak, J.D. (1998), Conocimiento y aprendizaje: Los mapas conceptuales como berramientas facilitadoras para escuelas y empresas, Alianza Editorial, Madrid.

Novak, J.D. (1990), Concept mapping: a useful tool for science education, Journal of Research in Science Teaching, Vol. 27, No. 10, pp. 937-949.

O’Donnell, A.M.; Dansereau, D.F.; Hall, R.H. (2002), Knowledge map as scaffolds for cognitive processing, Educational Psychology Review, No. 14, pp. 71-86.

O'Reilly, T. (2005), "What is Web 2.0", available at: <http://www. oreilly.com/go/web2> (accessed 5-5-2007).

OCLC (2004), "Análisis del entorno de 2003 por OCLC: Reconocimiento de patrones", available at: <http://www.oclc.org > (16-3-2011).

Pace, A. (2003), "Virtual Reference: What's in a Name?", Computers in Libraries, Vol. 23, No. 4, pp. 55-56.

Pinto, M., Doucet, A., Fernández-Ramos, A. (2010), "Measuring students' information skills through concept mapping", Journal of Information Science, Vol. 36, No. 4, pp. 464-80.

Pinto, M., Manso, R. (2012), "Virtual Reference Services: Are they evolving in line with current technological tendencies? An evaluation proposal", Electronic Library, Vol.29, No. 1, pp. 51-69.

Pomerantz, J. (2008), "Evaluation of Online Reference Services", Bulletin of the American Society for Information Science and Technology, Vol. 34, No. 2, pp. 15-19.

Pottier, P., et al. (2010), "Exploring how students think: a new method combining think-aloud and concept mapping protocols", Medical Education, Vol. 44, pp. 926-35.

Rojas Benitez, J. L. (2003), Diseño de Servicios de Información: Selección de Lecturas, Felix Varela, La Habana.

RUSA (2008), "Definitions of Reference", available at: http://www.ala. org/ala/mgrps/divs/rusa/archive/protools/referenceguide/definitionsreference.cfm (5-11-2011).

Shachaf, P., Horowitz, S. M. (2008), "Virtual reference service evaluation: Adherence to RUSA behavioral guidelines and IFLA digital reference guidelines", Library \& Information Science Research, Vol. 30, No. 2, pp. 122-37. 
Sherratt, C. S., Schlabach, M. L. (1990), "The Applications of Concept Mapping in Reference and Information Services", Reference Quarterly, Vol. 30, No. 1, pp. 60-69.

Tümen, S., Taspmar, M. (2007), "The effects of Concept Mapping on students' achievements in language teaching." Paper presented at the International Educational Technology (IETC) Conference 7th, Nicosia, Turkish Republic of Northern Cyprus, May, 3-10 2007.

Vargas Jiménez, S. (2010), "Los mapas conceptuales como herramienta de gestión de conocimiento en un servicio de referencia en ciencias de la salud: caso biblioteca Juan Roa Vásquez de la Universidad El Bosque", in J.; Cañas Sánchez, A.J.; Novak, J.D. (Ed.), Concept Maps: Making Learning Meaningful, Viña del Mar, Chile,

Weise, F. O., Borgendale, M. (1986), "EARS: Electronic Access to Reference Service", Bulletin of the Medical Library Association, Vol. 74, pp. 41-44.

White, M. D. (2001), "Digital reference services: Framework for analysis and evaluation", Library \& Information Science Research, Vol. 23, pp. 211-31.

Whitlatch, J. B. (2001), "Evaluating Reference Services in the Electronic Age", Library Trends, Vol. 50, No. 2, pp. 207-17.

Wong, G. (2010), "Information Commons Help Desk Transactions Study", The Journal of Academic Librarianship, Vol. 36, No. 3, pp. 235-241 